\title{
Loss of MTUS1 in gastric cancer promotes tumor growth and metastasis
}

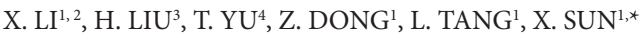 \\ ${ }^{1}$ Mental Health Center, West China Hospital, Sichuan University, Chengdu, 610041, China; ${ }^{2}$ Mental Health Center, First Affiliated Hospital, \\ Chongqing Medical University, Chongqing, 400016, China; ${ }^{3}$ Daping Hospital, Third Military Medical University, Chongqing, 400016, China; \\ ${ }^{4}$ Bio-X Institutes, Key Laboratory for the Genetics of Developmental and Neuropsychiatric Disorders (Ministry of Education), Shanghai Jiao \\ Tong University, Shanghai 200030, China
}

${ }^{*}$ Correspondence: xuelisun2009@126.com

Received March 10, 2013 / Accepted June 13, 2013

\begin{abstract}
Though the overall incidence of gastric cancer was decreasing in the developed countries in the past decades, it is still a serious threat to human health throughout the world. The molecular mechanisms underlying development of gastric cancer remains unclear. Though accumulating evidences shed a light on the implications of mitochondrial tumor suppressor (MTUS1) in carcinogenesis, the functional role of MTUS1 in regulation of proliferation and metastasis of gastric cancer cell is still poorly understood. In this study, we showed that the level of MTUS1 expression is relatively low in gastric cancer cell lines compared to normal gastric epithelial cells. By using clinical samples, we found that MTUS1 expression is downregulated in tumor tissues compared to non-cancerous counterpart, and loss of MTUS1 was associated with high incidence of lymph node metastasis and poor patient outcome. Moreover, we demonstrate that MTUS1 has a significant impact on both the proliferative and metastatic potential of gastric cancer cell line, which were further supported by using mice tumor xenograft models. The present data suggested MTUS1 as a potential tumor suppressor in gastric cancer and might lead to a better understanding of gastric carcinogenesis.
\end{abstract}

Key words: gastric cancer, MTUS1, proliferation, metastasis

Though the overall incidence of gastric cancer was decreasing in the developed countries in the past decades, it is still a serious threat to human health throughout the world [1]. Gastric cancer is responsible for the second most widely diagnosed malignancy with approximately 880000 new cases per year, and ranks as the second leading cause of cancer-related death worldwide [2]. Adenocarcinoma, which could be histologically subdivided into well-differentiated type and diffuse type, counts for nearly $90 \%$ gastric cancer [3] Surgical excision is currently the major method in treating gastric cancer, however, surgical options are often limited in most patients with advanced diseases. Though surgical excision combined with chemotherapy improves the therapeutic management of advanced gastric cancer, the usage of cytotoxic drugs result in severe side effects which lowers the life quality of patients. On the other hand, peritoneal metastasis, which evoked by direct cancer cell dissemination, is a frequent cause of death even after surgical excision. The 5-year survival rate of patients with peritoneal metastasis is only $2 \%$. [4, 5].

It has been established that development of gastric cancer is a multiple-steps process, starting with pre-malignant in situ lesions, and at last tumor cell migration into a near cavity or a distant organ.[6]. Similar to other tumor types, both environmental and genetic factors jointly influence the risk of development of gastric cancer [7]. To date, a cluster of bio-markers associated with clinicopathologic classification of gastric cancer patients has been described. For instance, a $\mathrm{G} \rightarrow \mathrm{T}$ nucleotide substitution was revealed in the exon 7 of E-cadherin in familial gastric cancer patients. This mutation results in an impaired gene product of E-cadherin and is associated with aggressive and poorly differentiated tumor type [8]. Upregulation of human epidermal growth factor receptor 2 (HER2) was found in up to $22 \%$ of gastric cancer patients, and this aided in predicting the patients who may benefit from a treatment with trastuzumab, a HER2 specific antibody.[9]. Further, aberrant regulation of Wnt signaling component, including Wnt-5a, Frizzled-7 and DKK-3, confers a proliferative and aggressive phenotype of gastric cancer cells $[10,11]$. Despite these reasonable advantages, association of these bio-markers with the progression of gastric cancer significantly varies in different areas and populations. Therefore, new targets for diagnostic and therapeutic management of gastric cancer are still urgently needed. 
The mitochondrial tumor suppressor (MTUS1) gene is mapped to the reverse strand of the chromosome 8p22, which is reported to be frequently deleted in tumors [12]. Recently, loss of MTUS1 was observed in several cancer types, including pancreatic cancer, ovary cancer, colorectal cancer and head-and-neck cancers [13]. Nevertheless, the implications of MTUS1 in gastric cancer are relatively unknown. In this study, we reported that MTUS1 was markedly downregulated in gastric cancer. Alteration of MTUS1 has a significant impact on proliferation and metastasis of gastric cancer cells.

\section{Materials and methods}

Clinical specimens. All gastric cancer tissues and corresponding adjacent non-cancerous tissues were obtained from Sichuan Provincial People's Hospital (Chengdu, China). Ethics approval was obtained from the Institutional Ethics Committee of Sichuan University, and informed consent for tissue procurement was obtained from all patients, or their relatives, before sampling. The stage of lymph node metastasis was determined according to the TNM classification system of the International Union against Cancer (UICC).

Cells culture. Human gastric epithelial cells were isolated from the biopsy tissue from superficial gastritis patients under informed consent, as described previously [14]. Human gastric cancer cell line SGC7901, MKN28, AGS, SUN16, MKN45, MKN74 were maintained in RPMI 1640 (Gibco, USA) containing $10 \%$ fetal calf serum (Gibco, USA), penicillin (100 U/L) and streptomycin $(10 \mathrm{mg} / \mathrm{L})$.

Transfection and establishing stable cell lines. Plasmids or siRNAs were transfected into SGC7901 cells using Lipofectamine 2000 (Invitrogen) according to the manufacturer's instructions. G418 (Amresco Inc.) at a concentration of 600 $\mu \mathrm{g} / \mathrm{ml}$ was used to select stable transfectants.

Reagents. Mouse-anti-MTUS1 was purchased from Abnova and mouse-anti- $\beta$-actin was purchased from Santa Cruz.

MTUS1 expression plasmid (pReceiver-M68-MTUS1) and mock vector (pReceiver-M68) were purchased from GeneCopoeia (Guangzhou, China).

Real-time RT-PCR. Total RNA was isolated from clinical tissues or cells using TRIzol reagent (Invitrogen, Carlsbad, CA, USA) according to the manufacturer's instructions. The real-time quantitative RT-PCR amplification of MTUS1 was performed with the ABI 7500 Real-Time PCR system using the SYBR-Green I kit (Takara Biotechnology, Dalian, China). The primers were synthesized following previous report [15].

Immunohistochemistry. The experimental protocol for immunohistochemical staining was described previously [16]. The immunoreactivity score, ranging from 0 to 16 , was measured as immunostaining intensity $(0$, negative; 1 , weak; 2 , moderate; 3 , strong; 4 ) multiplied by percentage of positive cells $(0,<5 \% ; 1,6-25 \% ; 2,26-50 \% ; 3,51-75 \% ; 4,>75 \%)$. The results were defined as: 0-4, low; 5-16, high. Any discrepancy between the two evaluators was resolved by reevaluation and careful discussion until agreement was reached.
Immunoblot. Total protein was extracted by using RIPA buffer and quantified by DC protein assay kit (Bio-Rad). After separation by $10-12 \%$ SDS-PAGE, the protein sample was transferred to PVDF membranes (Amersham Biosciences). The membranes were blocked for $1 \mathrm{~h}$ at $37^{\circ} \mathrm{C}$, and then incubated with primary antibodies at $4^{\circ} \mathrm{C}$ overnight. Membranes were subsequently probed with the secondary antibody (diluted 1:10,000; Santa Cruz Biotechnology) conjugated to horseradish peroxidase for $2 \mathrm{~h}$ at room temperature. Finally, the blots were visualized by enhanced chemiluminescence reagents (Amersham Biosciences).

MTT assay. MTT assay was performed using 96 -well plates. After treatment, $20 \mu \mathrm{l}$ MTT ( $5 \mathrm{mg} / \mathrm{ml}$, Sigma, St. Louis, MO) was added in each well and incubated for $2 \mathrm{~h}$. After removing the medium, the precipitate was dissolved in $150 \mu$ l Dimethyl Sulfoxide (DMSO, Amresco). The absorbance values were examined at $595 \mathrm{~nm}$ wavelength via a Spectra Max M5 (MDC, Sunnyrale, VA, USA).

Colony formation assay. Cells were seeded in six-well plate at a density of 500 cells per well. Cells were transfected with MTUS1 expression plasmid or mock vector for $48 \mathrm{~h}$, and then incubated with fresh medium for 14 days. Colonies were washed, fixed with methanol, stained with Crystal Violet (Sigma, St. Louis, MO, USA), and counted. Only those clones with more than 50 cells were considered as positive clones.

TUNEL assay. TUNEL assay was performed by using DeadEnd $^{\text {tu }}$ Fluorometric TUNEL System (Promega), following the manufacturer's instructions.

Scratch wound healing assay. Wounds were created in confluent cells by scraping the cell surface using a pipette tip. The cells were then washed for at least five times to remove free-floating cells. Cell motility was evaluated by counting the number of cells migrated into scraped area.

Cell invasion assay. Cell invasion was examined by using transwell chambers (Corning, USA). The bottom chamber was prepared with $10 \%$ FBS as a chemo-attractant. Matrigel (1:4, BD Biosciences, USA) was added to the transwell chambers and incubated at $37^{\circ} \mathrm{C}$ for $4 \mathrm{~h}$. Cells $\left(2.5 \times 10^{4}\right.$ cells per well $)$ were suspended in serum-free medium, and added into the upper chamber. After 48 hours, the number of cells, which migrated to the lower chamber was counted.

Mouse xenograft model. The experimental protocol for establishing mouse xenograft model was approved by the Institutional Animal Care and Treatment Committee of Sichuan University.

For tumor growth assay, SGC7901 sub-clones that stably expressed MTUS1 or mock vector were injected subcutaneously in nude mice $\left(2 \times 10^{6}\right.$ cells per mouse). Tumor volume was measured every five days, and mice were sacrificed 25 days after injection.

For lung metastasis model, SGC7901 sub-clones were suspended in PBS, and subsequently injected in nude mice $\left(1 \times 10^{6}\right.$ cells per mouse) through tail vein. Animals were sacrificed 45 days after injection. The lung weight and the number of 
A

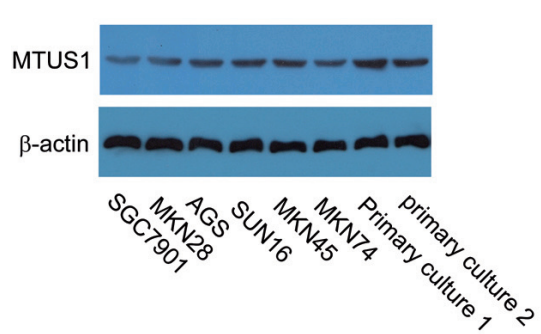

C

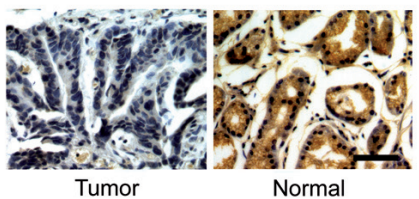

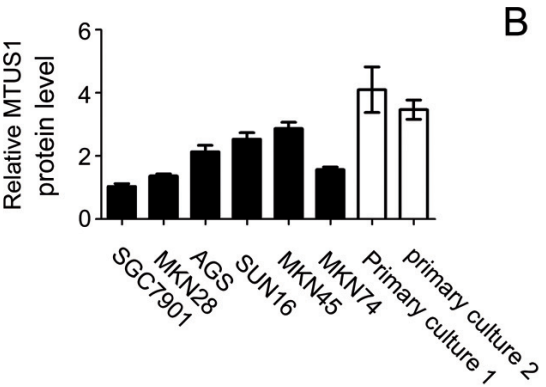

B
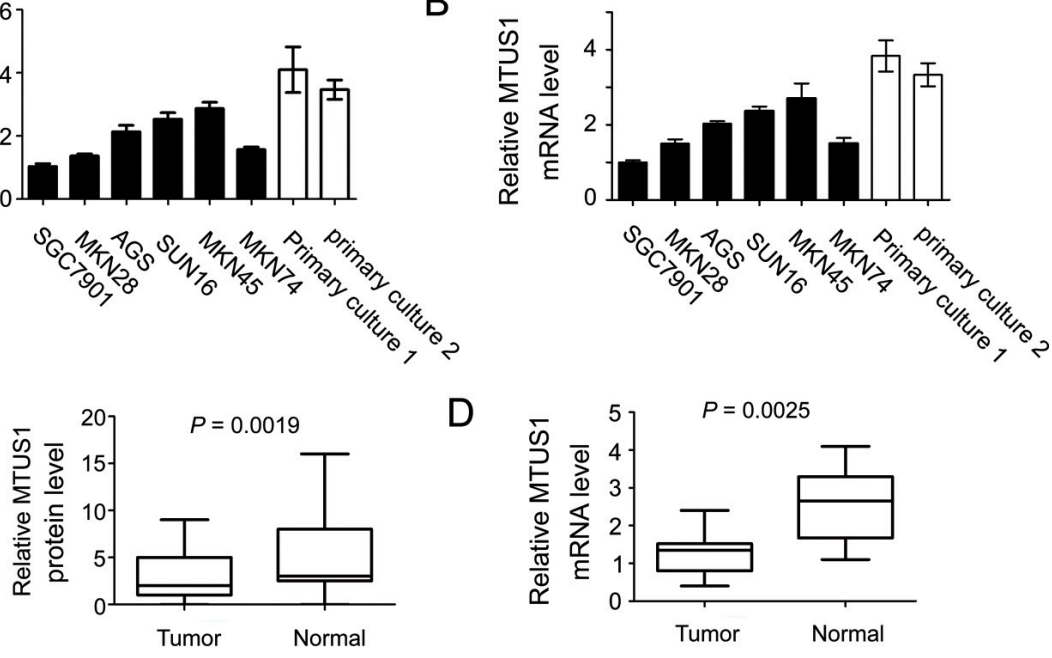

Figure 1. MTUS1 was down-regulated in gastric cancer

(A) MTUS1 expression in SGC7901, MKN28, AGS, SUN16, SKM45, MKN74 cell lines and two isolations of primary gastric epithelial cells was examined by immunoblot (left panel). $\beta$-actin was used as internal control. Each blotting strip was determined by Quality-One software, and the $\mathrm{x}$ axis shows the average intensity of three parallel experimental runs (right panel).

(B) MTUS1 expression in SGC7901, MKN28, AGS, SUN16, SKM45, MKN74 cell lines and two isolations of primary gastric epithelial cells was examined by real time RT-PCR.

(C) A total of 45 paired gastric cancer tissues and corresponding non-cancerous tissues were collected. Expression of MTUS1 in clinical samples was examined by immunostaining (left panel). Expression of MTUS1 in tumor tissues and non-cancerous corresponding tissues were compared based on the immunoreactivity score of each slide (right panel). Scale bar, $40 \mu \mathrm{m}$.

(D) Expression of MTUS1 in 10 paired gastric cancer tissues and corresponding non-cancerous tissues were examined by real time RT-PCR.

lung metastatic nodules were used as indices to evaluate lung metastatic capacity of cancer cells.

Five mice were used for each group in either tumor growth model or tumor metastasis model.

Statistics analysis. Differences between two groups were assessed by Student's $t$ test. ${ }^{*}, \mathrm{P}<0.05 ;{ }^{* *}, \mathrm{P}<0.01$; ${ }^{* * *}, \mathrm{P}<0.001$.

\section{Results}

Expression of MTUS1 is repressed in gastric cancer. As an initial test, we set out to determine the expression of MTUS1 in primary gastric epithelial cells and several gastric cancer cell lines, including SGC7901, MKN28, AGS, SUN16, MKN45 and MKN74. By both immunoblot and real time RT-PCR, both two isolations of primary gastric epithelial cells showed a strong MTUS1 expression. In contrast, a moderate level of MTUS1 expression was found in AGS and SUN16 cell lines, and a markedly low MTUS1 expression was observed in SGC7901, MKN28 and MKN74 cells (Fig. 1A-B).

To examine the clinical relevance of MTUS1 expression in gastric cancer, 45 paired gastric cancer tissues and corresponding non-cancerous tissues were collected. Expression of MTUS1 in clinical samples was evaluated by immunostaining. As show in Fig 1C, MTUS1 was markedly down-regulated
A

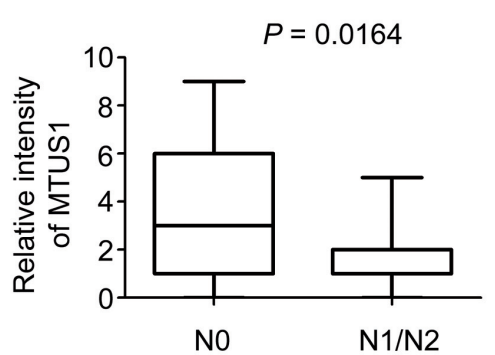

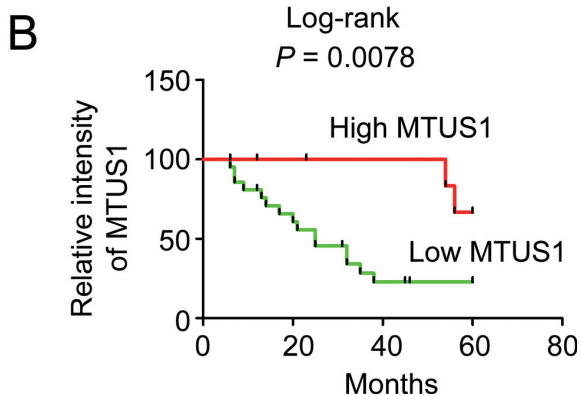

Figure 2. Loss of MTUS1 expression was associated with lymph node metastasis and poor overall survival rate (A) Expression of MTUS1 in the primary tumors without (N0) or with (N1/N2) lymph node metastasis was analyzed.

(B) Overall survival curves based on the MTUS1 expression in primary tumors was plotted by Kaplan-Meier survival analysis. 
in cancer tissues compared to non-cancerous tissues $(P=$ 0.0019). Low level of MTUS1 expression was observed in $73.3 \%(33 / 45)$ of gastric cancer samples, while high level of MTUS1 expression was found in only $26.7 \%$ (12/45) of gastric cancer samples. Correspondingly, the level of MTUS1 mRNA was also decreased in gastric cancer tissues compared to noncancerous tissues ( $P=0.0025$, Fig 1D).

Downregulation of MTUS1 is correlated with cancer metastasis and poor prognosis of gastric cancer. To explore whether MTUS1 expression is correlated with gastric cancer metastasis, MTUS1 expression was examined in 37 primary gastric cancer tissues with (N1/2) or without (N0) lymph nodes metastasis. As shown in Fig. 2A, low MTUS1 expression was more likely to be associated with lymph node metastasis $(P=$ $0.0164)$. Low MTUS1 expression was found in $61.9 \%(13 / 21)$ of samples at N0 stage versus $93.8 \%(15 / 16)$ of samples at N1/2 stages.

Further, Kaplan-Meier survival analysis was performed to determine the correlation between MTUS1 expression and patient prognosis. As results, the five-year overall survival rates of patients with low MTUS1 expression were significantly lower than those with high MTUS1 expression $(P=$ 0.0078 , Fig. $2 B$ ). Therefore, the represent data suggested that expression of MTUS1 was correlated with cancer metastasis and poor prognosis.

MTUS1 inhibits proliferation and induces apoptosis in gastric cancer cell line. To determine if MTUS1 has a role in regulating growth of gastric cancer cells, SGC7901 cell line was selected as an in vitro model. MTUS1 was overexpressed in SGC7901 cells by transiently transfection with an MTUS1 expression construct (Fig. 3A), and proliferation rate of SGC7901 was examined by MTT (3-(4, 5-dimethylthiazol-2yl)-2, 5-diphenyltetrazolium bromide) assay and clonogenic formation assays. As shown by MTT assay, exogenous MTUS1 expression attenuated SGC7901 cell proliferation in a timedependent manner. In spite of no significant difference at $24 \mathrm{~h}$ post-transfection, proliferation ratio of SGC7901 cells decreased by $51.25 \%(P<0.05)$ at $48 \mathrm{~h}$ and $51.43 \%(P<0.05)$ at $72 \mathrm{~h}$ upon MTUS1 expression, compared to mock group (Fig. 3B). In contrast, the proliferation ratio of SGC7901 cells was increased after siRNA-mediated MTUS1 knockdown (Fig. S1A-B). These observations were further supported by colony formation assay. The number of clones in the culture of MTUS1-expressed SGC7901 cells was 33.00 \pm 3.49 , while the number of clones was $81.25 \pm 4.87$ in mock control cells, with an inhibition ratio of $59.38 \%(P<0.001)$ (Fig. 3C). Since transient overexpression may not last too long time, stable SGC7901 cell lines were established (Fig. S2A). As shown in Fig. S2B, those SGC7901 cells stably transfected with MTUS1 expression plasmid formed clearly less number of clones compared to mock control cells $(P=0.0045)$.

To investigate if expression of MTUS1 induces cancer cell death, TUNEL staining was performed to measure the apoptosis in SGC7901 cells. Those cells with genome DNA breaks were labeled with free 3'-OH termini and visualized by fluorescence microscopy. As shown in Fig. 3D, a significantly increased number of TUNLE-positive cells were found in MTUS1-expressed SGC7901 cells (76.25 \pm 7.41$)$, compared to mock vector-treated cells $(32.75 \pm 3.50, P<$ $0.01)$. Summarizing, these results indicated that MTUS1 inhibits proliferation and induces apoptosis in gastric cancer cell line.
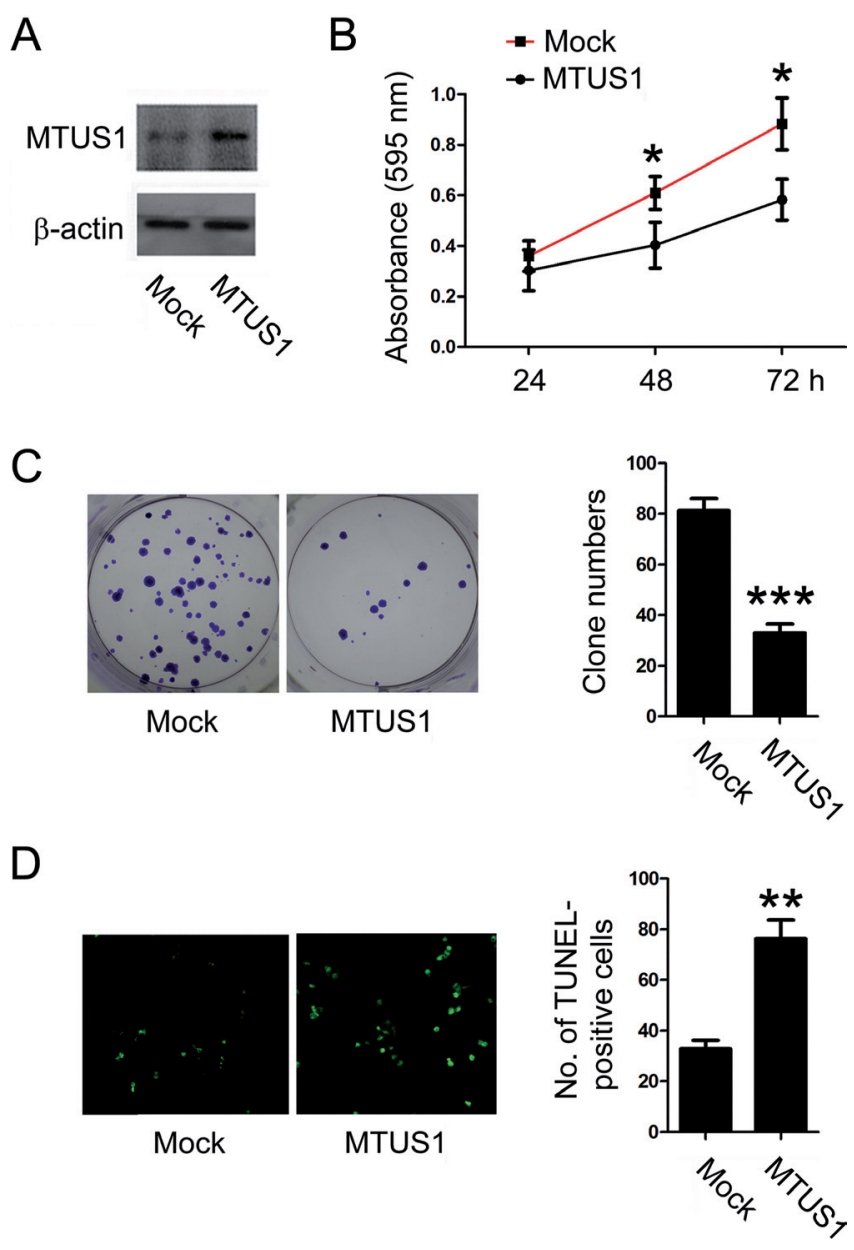

Figure 3. Overexpression of MTUS1 inhibited proliferation of gastric cancer cells

(A) SGC7901 cells were transfected with pReceiver-M68-MTUS1 plasmid or mock vector for $48 \mathrm{~h}$. Expression of MTUS1 in SGC7901 cells was examined by immunoblot.

(B) SGC7901 cells were transfected with pReceiver-M68-MTUS1 plasmid or mock vector for indicated time, proliferation of SGC7901 cells was examined by MTT assay.

(C) SGC7901 cells were transfected with pReceiver-M68-MTUS1 plasmid or mock vector for $48 \mathrm{~h}$, proliferation of SGC7901 cells was examined by colony formation assay.

(D) SGC7901 cells were transfected with pReceiver-M68- MTUS1 plasmid or mock vector for $72 \mathrm{~h}$, apoptosis of SGC7901 cells was examined by TUNEL assay.

All data were representative of three independent experiments. ${ }^{*}, \mathrm{P}<0.05$; ${ }^{* *}, \mathrm{P}<0.01 ;{ }^{* * *}, \mathrm{P}<0.001$. 
A

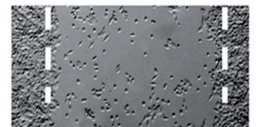

Mock

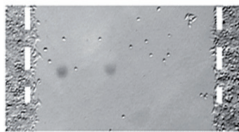

MTUS1

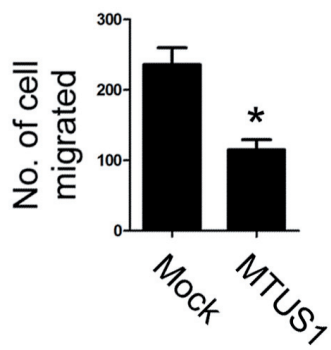

B

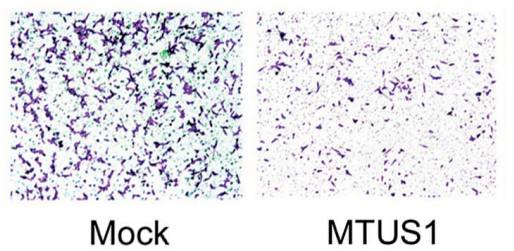

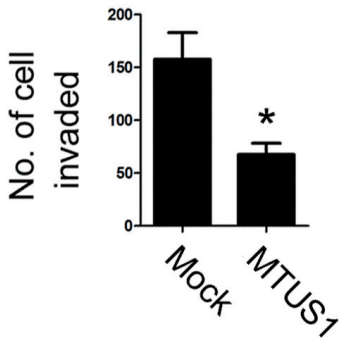

Figure 4. Overexpression of MTUS1 inhibited metastasis of gastric cancer cells

(A) SGC7901 cells were transfected with pReceiver-M68- MTUS1 plasmid or mock vector for 48 h. Cell migratory capability was examined by wound scratch assay.

(B) SGC7901 cells were transfected with pReceiver-M68- MTUS1 plasmid or mock vector for $48 \mathrm{~h}$. Cell invasive capability was examined by Matrigel assay. All data were representative of three independent experiments. ${ }^{*}, \mathbf{P}<0.05 ;{ }^{* *}, \mathbf{P}<0.01 ;{ }^{* *}, \mathbf{P}<0.001$.

MTUS1 limits metastasis of gastric cancer cells. Since MTUS1 expression is correlated with lymph node metastasis of gastric cancer, the impact of MTUS1 on migratory and invasive capability of gastric cancer cells were validated. In wound healing assay, massive SGC7901 cells transfected with mock vector were found to migrate into the wound areas $48 \mathrm{~h}$ after the wounds were created. In contrast, the number of migrated cells was markedly decreased in MTUS1-expressed SGC7901 cells $(P<0.05)$ (Fig. 4A). Similarly, in Matrigel invasion assay, the invasive capability of SGC7901 cells was substantially abolished by $57.10 \%(P<0.05)$, upon MTUS1 expression (Fig. 4B). In contrast, both migratory and invasive capability of SGC7901 cells was enhanced upon MTUS1 knockdown, compared to control cells (Fig. S3A-B). These results suggested that overexpression of MTUS1 limits the motility of these gastric cancer cells.

MTUS1 inhibits both growth and metastasis of gastric cancer cells in vivo. To further support our in vitro observations, tumor-bearing mouse models were utilized to verify the effect of MTUS1 on gastric cancer growth. The two sub-clones were subcutaneously transplanted in nude mice, respectively, and the volume of the tumor xenograft was measured every five days. As shown in Fig. 5A, no significant difference was found in tumor volume at 5 days post-transplantation. Notably, the tumors formed by MTUS1-overexpressed cancer cells exhibited a markedly lowered growth rate even 10 days post-transplantation. The average tumor volume decreased by $37.79 \%(P<0.01)$ at day $10,42.97(P<0.001)$ at day 15 , $55.90 \%(P<0.001)$ at day 20 and $41.17 \%(P<0.001)$ at day 25 in MTUS1-overexpressed group, compared to control group.
Next, SGC7901 sub-clones was injected in node mice through caudal vein, respectively, and dissemination of cancer cells to lung was examined. As shown in Fig. 5B, the SG7901 cell stably expressing MTUS1 exhibited clearly attenuated lung metastasis, revealed by both the decreased number of lung metastatic nodules $(P<0.001)$ and the reduced lung weight $(P<0.01)$. Therefore, the present data provided evidences that MTUS1 harbored both anti-proliferative and anti-metastatic effects on gastric cancer cells.

\section{Discussion}

Gastric cancer has been among the most common lifethreatening malignancies worldwide for decades, and, even nowadays, it is still a major problem in public health [1]. Development of gastric cancer is a multiple-step process, and results from complicated interconnections between genetic and environmental factors which regulate cell cycle, immune evasion, energy metabolism and cell-cell adhesive contacts [17]. Although it has long been established that Helicobacter pylori infection was responsible for over $80 \%$ incidence of gastric cancer, the poor diagnosis and high mortality of gastric cancer pointed out the insufficient understanding of its etiological factors and the lack of effective treatments [18]. Thus a more detailed identification of the key molecules involved in gastric cancer development must be amenable to both early diagnosis and discovery of new drug target.

Frequent loss of specific chromosomal regions in tumors suggested these regions as sites containing tumor suppressor genes which were probably repressed in tumors[19]. 
A
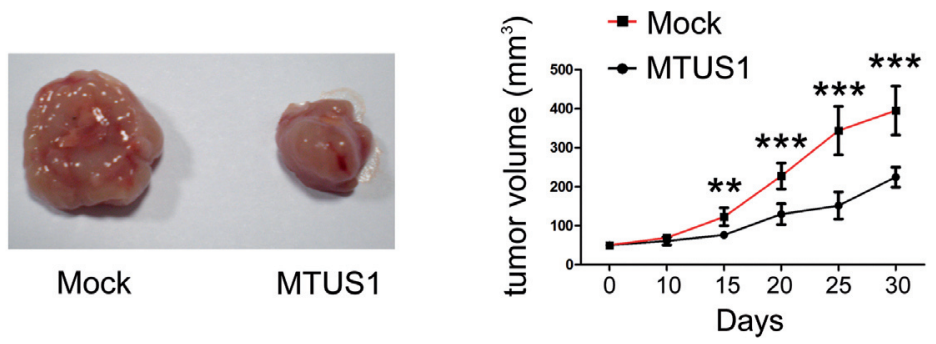

B
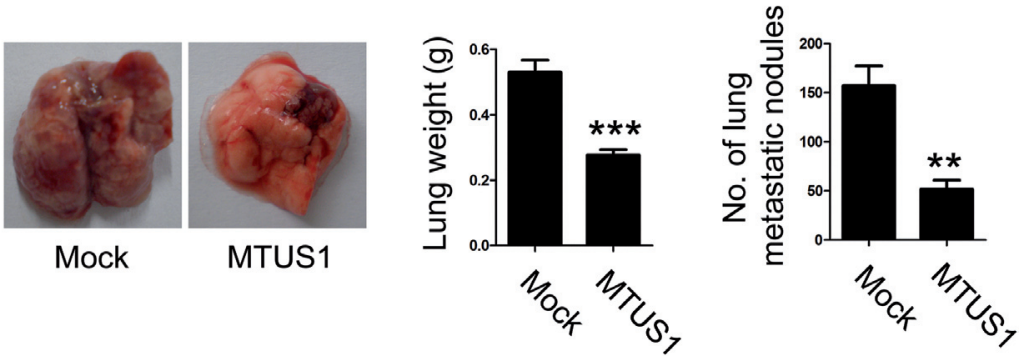

Figure 5. MTUS1 limited gastric cancer growth and metastasis in vivo

(A) SGC7901 cells that were stably transfected with pReceiver-M68-MTUS1 plasmid or mock vector were subcutaneously transplanted in nude mice $(n=5)$. Representative image of tumors from MTUS1-expressed group or mock control group was shown (left panel). The tumor volume was measured every five days (right panel).

(B) SGC7901 cells that were stably transfected with pReceiver-M68-MTUS1 plasmid or mock vector were intravenously injected in nude mice (n = 5). Representative images of lungs from MTUS1-expressed group or mock control group was shown (left panel). Cancer cell metastasis was examined by measuring the number of lung metastatic nodules (middle panel) or evaluating the lung weight (right panel).

All data were representative of three independent experiments. ${ }^{*}, \mathrm{P}<0.05 ;{ }^{* *}, \mathrm{P}<0.01{ }^{* \star *}, \mathrm{P}<0.001$

Chromosome 8p22 was reported as a hotspot for chromosome deletion in several tumor types, and the implications of those genes mapped to this region in tumor development have been continuously studied [20]. It is reported that expression of FEZ1 was depressed in many tumors with 8 p22 deletions, including prostate cancer, esophageal cancer and gastric cancer. Fez1 played crucial roles in limiting cancer cell proliferation by regulating mitosis [21]. Additionally, expression of another gene located in $8 \mathrm{p} 22$, Vps37A, was reduced in ovarian tumors, and downregulation of Vps37A was tightly associated with the reduced overall survival rate. Furthermore, the Vps37A-silenced cell lines exhibited resistance against Cetuximab treatment, suggesting a role of Vps37 A in chemo-resistance[22]. In this study, we showed that expression of MTUS1 was reduced in several gastric cancer cell lines compared to primary culture of gastric epithelial cells. Further, we demonstrated that these in vitro observations were of clinical relevance. Expression of MTUS1 was markedly repressed in gastric tumor tissues compared to non-cancerous counterparts. Further, low level of MTUS1 expression in primary tumor was associated with high incidence of lymph node metastasis and poor patient outcome.

Genetic abnormalities of MTUS1 have long been associated with cancer development. It is reported that, by searching in 17 coding exons of MTUS1 in 51 hepatocellular carcinoma
(HCC) tumors and 58 HCC cell lines, five major nucleotide substitutions were identified. Loss of heterozygosity (LOH) caused amino acid substitution, deletion of conserved structural motifs or dys-regulated RNA splicing, leading to potential deleterious effects on MTUS1 [23]. Further, copy number variations (CNV) of MTUS1 were also found to be associated with the risk for familial breast cancer. It is documented that a $1.1 \mathrm{~kb}$ deletion in MTUS1 was implicated with a decreased risk of familial breast cancer. Nucleotide sequencing reveals a 41 bp stretch of homology flanking the exon [24]. Therefore, in spite of the present data, more work is still needed to determine whether loss of MTUS1 expression in gastric cancer is due to chromosome 8 p22 depletion or other mechanisms.

Though accumulating evidences shed a light on the implications of MTUS1 in carcinogenesis, the functional role of MTUS1 in regulation of proliferation and metastasis of gastric cancer cell is still poorly understood $[25,26]$. To this end, expression of MTUS1 was modulated in gastric cancer cell line SGC7901, and the proliferative and metastatic potential of these cells were compared. We demonstrated that overexpression of MTUS1 significantly decreased proliferation rate, triggered apoptotic cell death, and reduced migratory and invasive capability of SGC7901 cells. In contrast, knockdown of MTUS1 markedly increased both proliferation and metastasis of SGC7901 cells. Further, by using tumor xenograft models, we showed that overexpression of MTUS1 limited both sub- 
cutaneous tumor growth and lung-tropic metastasis. These results provided strong evidences indicating that MTUS1 was a potential tumor suppressor in gastric cancer. Our data are consistent with previous reports in breast cancer that silencing MTUS1 expression by siRNA facilitated breast cancer cell proliferation by accelerating cell cycle, and also promoted cell motility by regulating microtubule dynamics [27]. Further work will be conducted to illustrate if MTUS1 has a role in regulating tumor-associated angiogenesis or resistance to chemo-therapeutic drugs.

Summarizing, the present data represents new insights for the biological role of MTUS1 in the development of gastric cancer. Hopefully, this study also suggests MTUS1 as a potential target in treating gastric cancer.

Supplementary information is available in the online version of the paper.

Acknowledgements: This study was supported by grants from Key Research Project of Ministry of Education in China (No.308032).

\section{References}

[1] POLK DB, PEEK RM. Helicobacter pylori: gastric cancer and beyond. Nature Reviews Cancer 2010; 10: 403-14. http:// dx.doi.org/10.1038/nrc2857

[2] TSUGANE S, SASAZUKIS. Diet and the risk of gastric cancer: review of epidemiological evidence. Gastric Cancer 2007; 10: 75-83. http://dx.doi.org/10.1007/s10120-007-0420-0

[3] YOSHIDA T, ONO H, KUCHIBA A, SAEKI N, SAKAMOTO $\mathrm{H}$. Genome - wide germline analyses on cancer susceptibility and GeMDBJ database: Gastric cancer as an example. Cancer science 2010; 101: 1582-9. http://dx.doi.org/10.1111/j.13497006.2010.01590.x

[4] BANDO E, YONEMURA Y, TAKESHITA Y, TANIGUCHI $\mathrm{K}$, YASUI $\mathrm{T}$, et al. Intraoperative lavage for cytological examination in 1,297 patients with gastric carcinoma. American journal of surgery 1999; 178: 256. http://dx.doi.org/10.1016/ S0002-9610(99)00162-2

[5] SASAKO M, SANO T, YAMAMOTO S, KUROKAWA Y, NASHIMOTO A, et al. D2 lymphadenectomy alone or with para-aortic nodal dissection for gastric cancer. New England Journal of Medicine 2008; 359: 453-62. http://dx.doi. org/10.1056/NEJMoa0707035

[6] GONZALEZCA, AGUDO A. Carcinogenesis, prevention and early detection of gastric cancer: Where we are and where we should go. International Journal of Cancer 2012; 130: 745-53. http://dx.doi.org/10.1002/ijc.26430

[7] XIONG P, LI YX, TANG YT, CHEN HG. Proteomic Analyses of Sirt1-Mediated Cisplatin Resistance in OSCC Cell Line. The Protein Journal 2011;30: 499-508. http://dx.doi.org/10.1007/ s10930-011-9354-9

[8] GAYTHER SA, GORRINGE KL, RAMUS SJ, HUNTSMAN $\mathrm{D}$, ROVIELLO F, et al. Identification of germ-line E-cadherin mutations in gastric cancer families of European origin. Cancer research 1998; 58: 4086-9.
[9] GARCiA I, VIZOSO F, MARTiN A, SANZ L, ABDEL-LAH $\mathrm{O}$, et al. Clinical significance of the epidermal growth factor receptor and HER2 receptor in resectable gastric cancer. Annals of surgical oncology 2003; 10: 234-41. http://dx.doi. org/10.1245/ASO.2003.05.010

[10] KURAYOSHI M, OUE N, YAMAMOTO H, KISHIDA M INOUE A, et al. Expression of Wnt-5a is correlated with aggressiveness of gastric cancer by stimulating cell migration and invasion. Cancer research 2006; 66: 10439-48. http://dx.doi. org/10.1158/0008-5472.CAN-06-2359

[11] KIRIKOSHI H, SEKIHARA H, KATOH M. Up-regulation of Frizzled-7 (FZD7) in human gastric cancer. International journal of oncology 2001; 19: 111.

[12] DI BM, BIECHE I, DESHAYES F, VACHER S, NOUET $S$, et al. Structural organization and expression of human MTUS1, a candidate 8p22 tumor suppressor gene encoding a family of angiotensin II AT2 receptor-interacting proteins, ATIP. Gene 2006; 380: 127-36. http://dx.doi.org/10.1016/ j.gene.2006.05.021

[13] RODRIGUES-FERREIRA S, DI TOMMASO A, DIMITROV A, CAZAUBON S, GRUEL N, et al. 8p22 MTUS1 gene product ATIP3 is a novel anti-mitotic protein underexpressed in invasive breast carcinoma of poor prognosis. PloS one. 2009; 4: e7239. http://dx.doi.org/10.1371/journal.pone.0007239

[14] SMOOT D, RESAU J, NAAB T, DESBORDES B, GILLIAM T, et al. Adherence of Helicobacter pylori to cultured human gastric epithelial cells. Infection and immunity. 1993; 61: 350-5.

[15] YE H, PUNGPRAVAT N, HUANG B-L, MUZIO LL, MARIGGIÒ MA, et al. Genomic assessments of the frequent loss of heterozygosity region on 8p21.3 p22 in head and neck squamous cell carcinoma. Cancer genetics and cytogenetics 2007; 176: 100-6. http://dx.doi.org/10.1016/j.cancergencyto. 2007.04.003

[16] LI Z, ZHAO X, BAI S, WANG Z, CHEN L, et al. Proteomics identification of cyclophilin a as a potential prognostic factor and therapeutic target in endometrial carcinoma. Molecular \& cellular proteomics 2008; 7: 1810-23. http://dx.doi. org/10.1074/mcp.M700544-MCP200

[17] XU AG, LI SG, LIU JH, GAN AH. Function of apoptosis and expression of the proteins Bcl-2, p53 and C-myc in the development of gastric cancer. Apoptosis 2001; 17: 6 .

[18] UEMURA N, OKAMOTO S, YAMAMOTO S, MATSUMURA N, YAMAGUCHI S, et al. Helicobacter pylori infection and the development of gastric cancer. New England Journal of Medicine 2001; 345: 784-9. http://dx.doi.org/10.1056/ NEJMoa001999

[19] CAREN H, KRYH H, NETHANDER M, SJOBERG RM, TRÄGER C, et al. High-risk neuroblastoma tumors with 11q-deletion display a poor prognostic, chromosome instability phenotype with later onset. Proceedings of the National Academy of Sciences 2010; 107: 4323-8. http://dx.doi.org/ 10.1073/pnas.0910684107

[20] EL AT, BRUCHMANN M, ZUSTIN J, ISBARN H, HELLWINKEL OJ, et al. Chromosome $8 \mathrm{p}$ deletions and $8 \mathrm{q}$ gains are associated with tumor progression and poor prognosis in prostate cancer. Clinical Cancer Research 2010; 16: 56-64. http://dx.doi.org/10.1158/1078-0432.CCR-09-1423 
[21] CALIFANO D, PIGNATA S, PISANO C, GREGGI S, LAURELLI G, et al. FEZ1/LZTS1 protein expression in ovarian cancer. Journal of cellular physiology 2010; 222: 382-6. http:// dx.doi.org/10.1002/jcp.21962

[22] WITTINGER M, VANHARA P, EL-GAZZAR A, SAVARESEBRENNER B, PILS D, et al. hVps37A status affects prognosis and cetuximab sensitivity in ovarian cancer. Clinical Cancer Research 2011; 17: 7816-27. http://dx.doi.org/10.1158/10780432.CCR-11-0408

[23] DI BM, PINEAU P, NOUET S, BERHOUET S, SEITZ I, et al. Mutation analysis of the $8 \mathrm{p} 22$ candidate tumor suppressor gene ATIP/MTUS1 in hepatocellular carcinoma. Molecular and cellular endocrinology 2006; 252: 207-15. http://dx.doi. org/10.1016/j.mce.2006.03.014

[24] FRANK B, BERMEJO JL, HEMMINKI K, SUTTER C, WAPPENSCHMIDT B, et al. Copy number variant in the candidate tumor suppressor gene MTUS1 and familial breast cancer risk.
Carcinogenesis 2007; 28: 1442-5. http://dx.doi.org/10.1093/ carcin/bgm 033

[25] LOUIS SN, CHOW L, REZMANN L, KREZEL MA, CATT KJ, et al. Expression and function of ATIP/MTUS1 in human prostate cancer cell lines. The Prostate 2010; 70: 1563-74. http://dx.doi.org/10.1002/pros.21192

[26] DING X, ZHANG N, CAI Y, LI S, ZHENG C, et al. Downregulation of tumor suppressor MTUS1/ATIP is associated with enhanced proliferation, poor differentiation and poor prognosis in oral tongue squamous cell carcinoma. Molecular Oncology 2011; 6: 73-80. http://dx.doi.org/10.1016/j.molonc.2011.11.002

[27] MOLINA A, VELOT L, GHOUINEM L, ABDELKARIM M, BOUCHET BP, et al. ATIP3, a novel prognostic marker of breast cancer patient survival, limits cancer cell migration and slows metastatic progression by regulating microtubule dynamics. Cancer research 2013; 73: 2905-2915. http://dx.doi. org/10.1158/0008-5472.CAN-12-3565 


\title{
Supplementary Information
}

\section{Loss of MTUS1 in gastric cancer promotes tumor growth and metastasis}

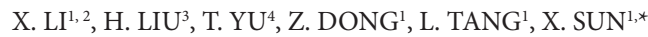

${ }^{1}$ Mental Health Center, West China Hospital, Sichuan University, Chengdu, 610041, China; ${ }^{2}$ Mental Health Center, First Affiliated Hospital, Chongqing Medical University, Chongqing, 400016, China; ${ }^{3}$ Daping Hospital, Third Military Medical University, Chongqing, 400016, China; ${ }^{4}$ Bio-X Institutes, Key Laboratory for the Genetics of Developmental and Neuropsychiatric Disorders (Ministry of Education), Shanghai Jiao Tong University, Shanghai 200030, China

*Correspondence: xuelisun2009@126.com

Supplementary Figures

A

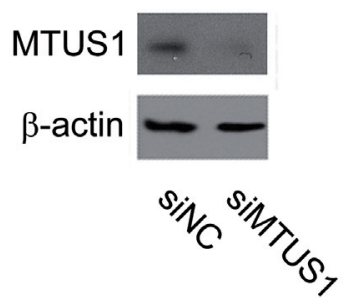

B

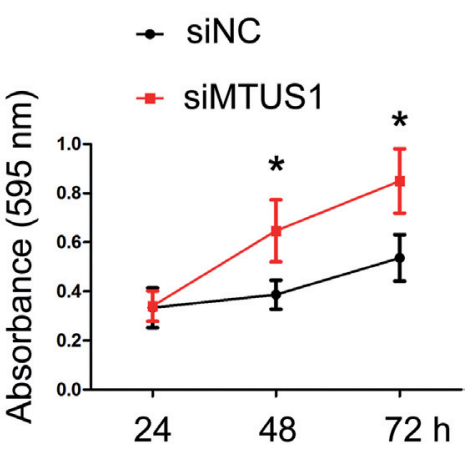

Figure S1. Knockdown of MTUS1 promoted proliferation of gastric cancer cells

(A) SGC7901 cells were transfected with siMTUS1 or negative control vector (siNC). Expression of MTUS1 was examined by immunoblot.

(B) Proliferation of SGC7901 cells was examined by MTT assay. 
A

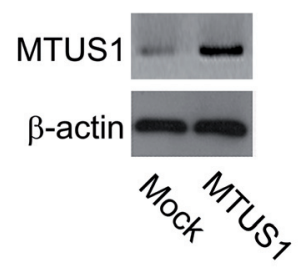

B
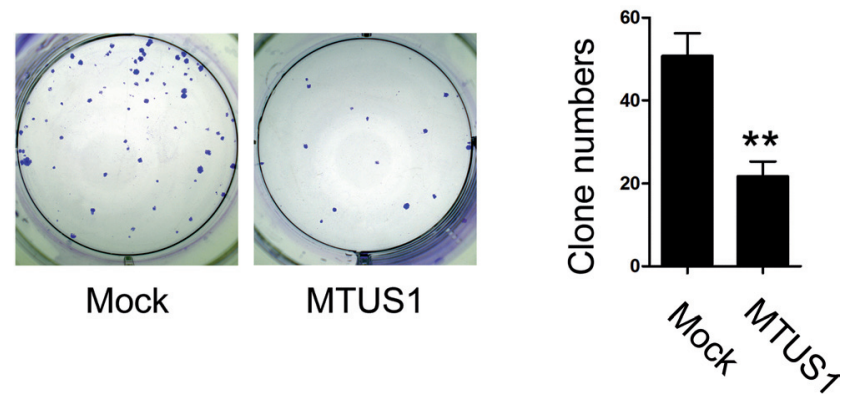

Figure S2. Overexpression of MTUS1 inhibited proliferation of gastric cancer cells

(A) SGC7901 cells were stably transfected with pReceiver-M68-MTUS1 plasmid or mock vector. Expression of MTUS1 was examined by immunoblot. (B) Proliferation of SGC7901 stable cell lines was examined by colony formation assay.

A

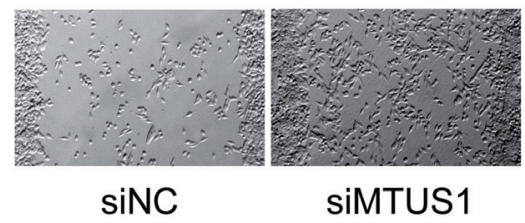

B

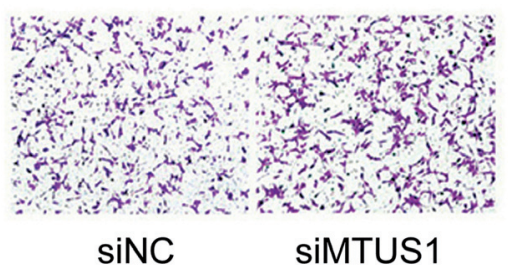

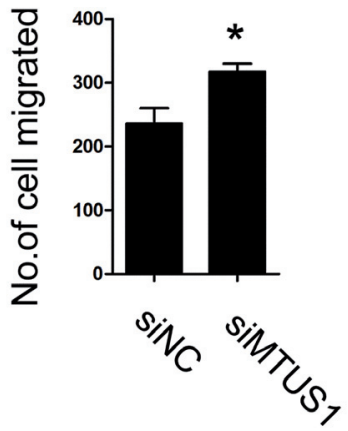

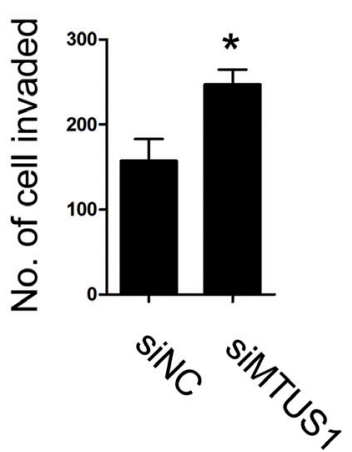

Figure S3. Repression of MTUS1 enhanced motility of gastric cancer cells.

(A) SGC7901 cells were transfected with siNC or siMTUS1. Cell migratory capability was examined by wound scratch assay.

(B) Cell invasive capability was examined by Matrigel assay. 